\title{
Morphometric studies of age related changes in normal human breast and their significance for evolution of mammary cancer
}

\author{
SW HUTSON, PN COWEN, CC BIRD \\ From the Institute of Pathology, University of Leeds, Leeds
}

SUMMARY Ageing changes in the normal human female breast were studied to determine their significance for the evolution of mammary cancer. Employing the morphometric techniques of point counting and planimetry, objective quantitative measurements were made of the structure of the normal female breast in 58 subjects from the prepubertal to late postreproductive period. The relative amounts of epithelial and connective tissue varied with age, and the epithelial elements (combined lobular and extralobular) were unevenly distributed within the gland, with lower containing more than upper quadrants. The upper outer quadrant, however, usually contained the largest proportion of lobular units, which may relate to the higher incidence of lobular carcinoma found in this quadrant. Involution was shown to be a premenopausal rather than postmenopausal phenomenon. Mammary dysplastic changes were uncommon in all age groups.

Normal human breast morphology has been well documented at both light and electron microscopical levels. ${ }^{1-8}$ Similar studies have also been conducted in breasts containing clinical carcinomas in order to determine early malignant and premalignant changes. $^{9-11}$ The extent to which normal ageing processes contribute to these changes is uncertain, however, since these are poorly documented. The present study was undertaken to establish on a quantitative basis the normal sequence of morphological changes that take place in the non-neoplastic female breast from the prereproductive to postreproductive periods. Particular attention was devoted to determining features that might have relevance for the subsequent evolution of breast cancer.

\section{Material and methods}

\section{BREAST SAMPLES}

Subcutaneously removed mammary glands, excluding the nipple and axillary tail, were routinely collected from female subjects at necropsy. The breasts were fixed in buffered formalin for three to seven days depending on size and fat content. In all, 58 breasts were processed covering the seven decades from 10 to 80 years, and each group contained at least eight samples. No bilateral specimens were taken.

\section{PREPARATION FOR SUBGROSS ANALYSIS}

After fixation, breasts were sliced coronally $(2 \mathrm{~mm}$ slices) on an Excel Boston slicing machine and labelled on their superior and lateral extremities to aid orientation. Where necessary, fixation was continued for a further $24 \mathrm{~h}$. Whole slices were stained with Harris's haematoxylin, dehydrated in graded ethanol $(70 \%, 95 \%$, and $100 \%)$, and cleared and stored in methyl salicylate. ${ }^{12}$

\section{PREPARATION FOR LIGHT MICROSCOPY}

After qualitative and quantitative examination at the subgross level tissue blocks were removed for histology. Twenty blocks (five from each quadrant), each measuring $1.5 \times 1.5 \times 0.2 \mathrm{~cm}$, were selected at random from five alternate slices. Before processing for light microscopy blocks were washed in several changes of absolute alcohol over a period of three days to remove all traces of methyl salicylate. After processing for routine histology $8 \mu \mathrm{m}$ sections were stained with haematoxylin and eosin and examined by light microscopy.

\section{MORPHOMETRY}

Volume density estimates using point counting

The volume density or volume proportion $\left(V_{v}\right)$ of a 
component may be estimated from the fractional area $\left(A_{A}\right)$ it occupies in a section plane. ${ }^{13}$ Several methods are available for determining $A_{A}$, but the most efficient is point counting. In this method a test system of points is superimposed on a section containing the structures to be measured. The fraction of test points falling within the structure is known as the point density $\left(\mathrm{P}_{\mathrm{P}}\right)$, and $\mathrm{V}_{\mathrm{v}}=\mathrm{A}_{\mathrm{A}}=\mathrm{P}_{\mathrm{P}} \mathrm{P}^{14}$ is

In the present study point counting was used to estimate the volume proportion of: (a) total epithelial tissue (combined lobular and extralobular) per quadrant; (b) epithelial and connective tissue components within the average lobe per quadrant; and (c) epithelial and connective tissue components within the average lobule per whole gland.

To estimate the amount of epithelial tissue in each quadrant, whole breast slices were placed in a large petri dish on to which had been etched calibrated crossed axes. The markings on the upper and outer extremities of breast slices were then aligned with these axes so as to divide the slice into quadrants (upper outer, upper inner, lower outer, and lower inner). Five areas within each quadrant were examined at a magnification of about $10 \times$ using a dissecting microscope (Kyowa) which contained a 36 point square lattice in one eyepiece. The first area examined in each quadrant was chosen at random using tables of paired random numbers ${ }^{16}$ to select the $\mathrm{X}$ and $\mathrm{Y}$ coordinates; the remaining four fields were arranged around the first in a square pattern. The volume proportion of a particular component $\left(\mathrm{V}_{\mathrm{v}}\right.$ component) is the ratio of the total number of points falling on the component ( $\mathrm{P}_{\mathrm{P}}$ component) to the total number of points applied $\left(P_{P}\right.$ total $)$.

$\mathrm{V}_{\mathrm{v}}$ component $=\frac{\mathrm{P}_{\mathrm{P}} \text { component } \times 100}{\mathrm{P}_{\mathrm{P}} \text { total }}($ equation 1$)$

Using the above formula the amount of epithelial tissue in each quadrant and hence its distribution within the whole gland was assessed.

The volume proportion of epithelial and connective tissue components of the average lobe and lobule were estimated from histological sections stained with haematoxylin and eosin. The magnifications used were roughly $63 \times$ and $160 \times$. Twenty four to 28 sections from each specimen were examined for the lobe analysis and between 20 and 28 lobules per breast specimen were examined to determine their average composition. In this analysis the microscope used contained a 100 point Lennox type lattice (Graticules Ltd) in one eyepiece. The volume proportion of the epithelial and connective tissue components was calculated using equation 1 .

\section{PLANIMETRY}

Histological sections were also used to estimate size and shape characteristics of the average lobule. Light microscopical images at a magnification of about $63 \times$ were projected on to the digitising tablet of a Kontron IBAS I semiautomatic analyser. The outlines of between 400 and 500 breast lobules per specimen were traced using a light cursor. This arrangement enabled a number of parameters to be measured accurately and rapidly from a single tracing for the storage and computation of results as required. The parameters presented in this study include diameter and volume, assuming the lobules to be spherical, and two shape factors $\mathrm{F}_{\mathrm{PE}}$ and $\mathrm{F}_{\mathrm{ELL}}$ were derived where:

$$
\begin{gathered}
F_{P E}=\frac{4 \times \text { area }}{\text { perimeter }} \\
F_{E L L}=\frac{\text { short diameter }}{\text { long diameter }}
\end{gathered}
$$

EFFECT OF PARITY AND MENSTRUAL STATE We attempted to correlate the amounts of epithelial tissue within each age group with parity and menstrual state. Reliable information on the latter could be obtained for only 45 of the 58 subjects. They were classed as having never been pregnant (nonparous) or having had one or more pregnancies (parous) and whether they were pre-, peri-, or postmenopausal. It was not possible to obtain accurate information about the stage of the menstrual cycle of premenopausal subjects.

\section{PRESENTATION OF RESULTS}

Data were collected for individual breast samples and the mean values together with the standard deviations calculated for each age group. The results are presented in graphical form.

\section{Results}

OVERALL BREAST STRUCTURE

Subgross and light microscopical analysis confirmed conventional views of the overall structure of the mammary gland. The glandular elements were contained within about 20 ill defined lobes each emptying through a separate terminal duct at the nipple. The average lobe consisted of a variable number of lobules and associated extralobular ducts supported by fibrous and fatty connective tissue. The shape of individual lobes is not clear as close packing renders their boundaries indistinct and in the coronal sections used in this study it was not possible to distinguish between adjacent lobes. The lobules themselves were made up of a variable number of acini or 


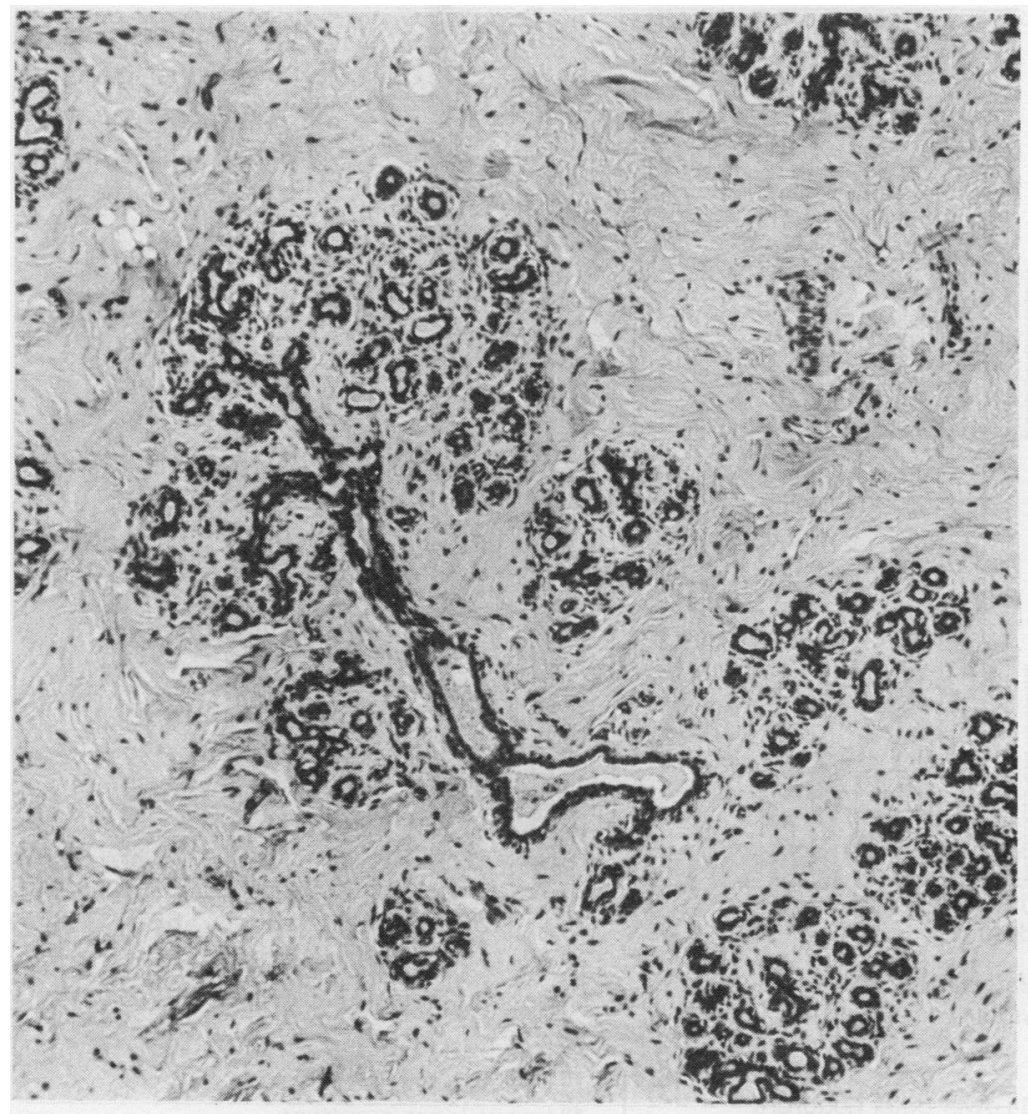

Fig. 1 Lobe of normal non-lactating breast showing lobules in fibro-connective tissue with an intralobular duct in the centre. Haematoxylin and eosin. $\times 92$

blind ductules arising from a branched intralobular duct (Fig. 1); the whole lobule was supported by loose fibrous connective tissue. Fatty tissue was not evident within the lobular unit. Accurate measurement of numbers of lobules and acini in each lobe was precluded by variations in their size and shape, their non-random distribution and orientation, and, most important, the pronounced paucity of lobules and acini in the older age groups.

Remarkably, few changes of mammary dysplasia were evident and no premalignant or frankly malignant changes were seen in any of the breasts examined. Sclerosed lobules were seen in only five subjects; two in the second decade, two in the third decade, and one in a 40 year old woman. Duct ectasia of at least slight degree was seen in 20 subjects but had no particular age association, although it appeared less common in teenagers. Only three breasts (from patients aged 13, 15, and 39 years) showed convincing evidence of lobular unfolding. " In contrast, microcysts developing from lobules were seen in most breasts, although they were fewer and smaller in the youngest age groups. In nine of these cysts microscopic papillary epithelial ingrowth into the cyst lumena was seen. This feature had no special age predilection. The smallest cysts blended, in size, into slightly ectatic ducts and it was impossible to decide at what level they represented a pathological change.

\section{DISTRIBUTION OF EPITHELIAL TISSUE BETWEEN QUADRANTS}

In general the lower half of the breast contained more epithelial tissue than the upper half (Fig. 2). In all quadrants the maximum amount of epithelial tissue was attained during the third decade with subsequent values falling rapidly until the sixth decade. Thereafter values either increased slightly or remained fairly constant (Fig. 2).

\section{COMPOSITION OF AVERAGE LOBE}

As already indicated, individual lobes could not be identified in coronal sections. In each quadrant, however, a region known as the lobe area, extending 


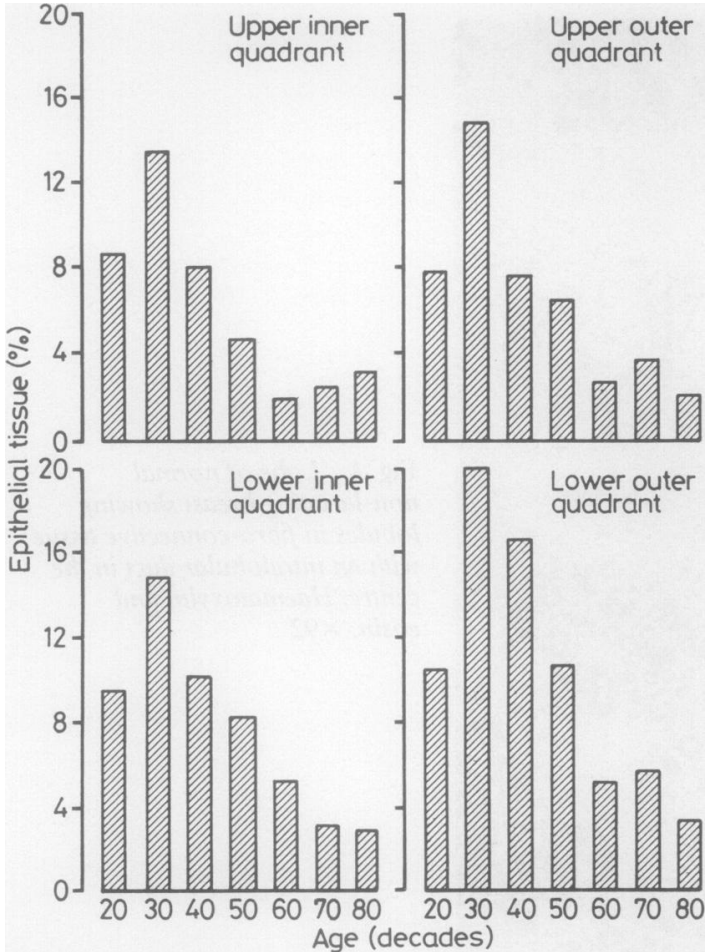

Fig. 2 Distribution of total epithelial tissue between the four breast quadrants as assessed by subgross morphometry.

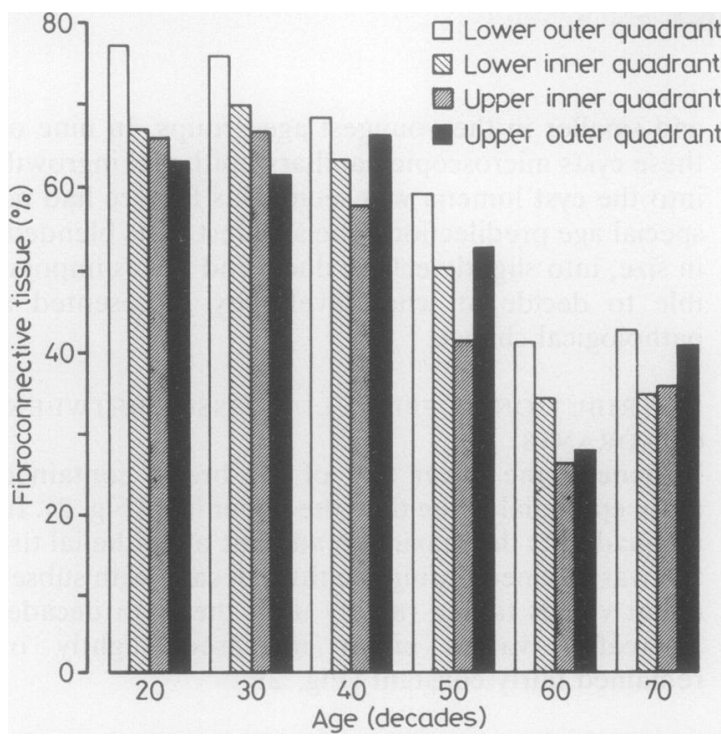

Fig. 4 Fibro-connective tissue content of average lobe in each quadrant with age.

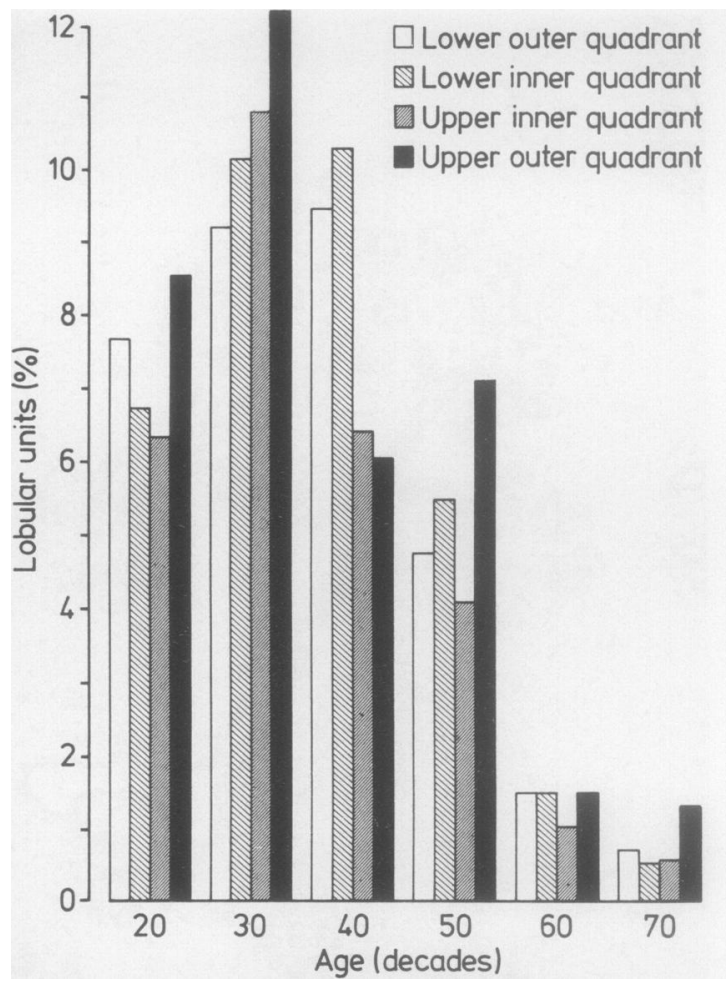

Fig. 3 Lobular composition of average lobe in each quadrant with age. ${ }^{*}$

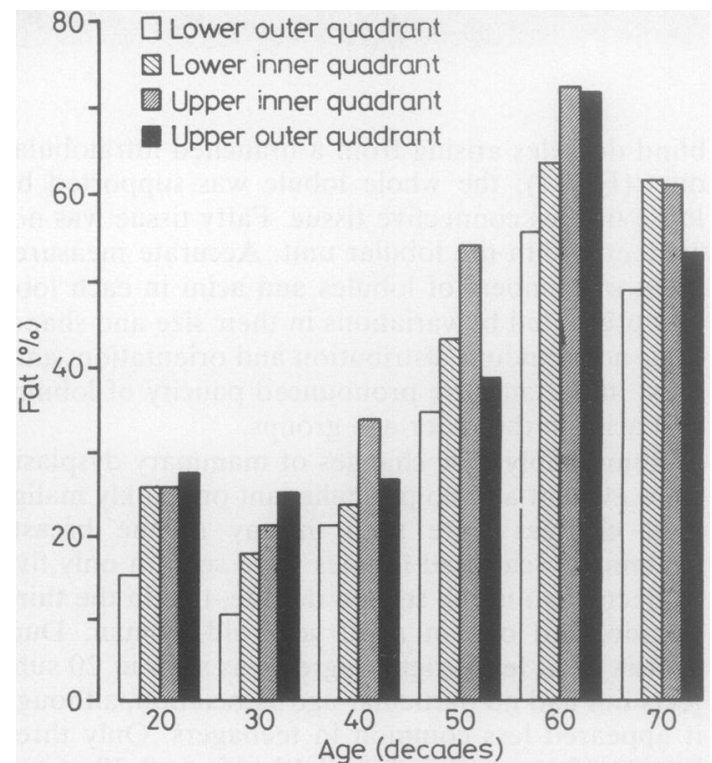

Fig. 5 Fat content of average lobe in each quadrant with age.

${ }^{*}$ Figs. 3-8 show assessments based on histological morphometry. 


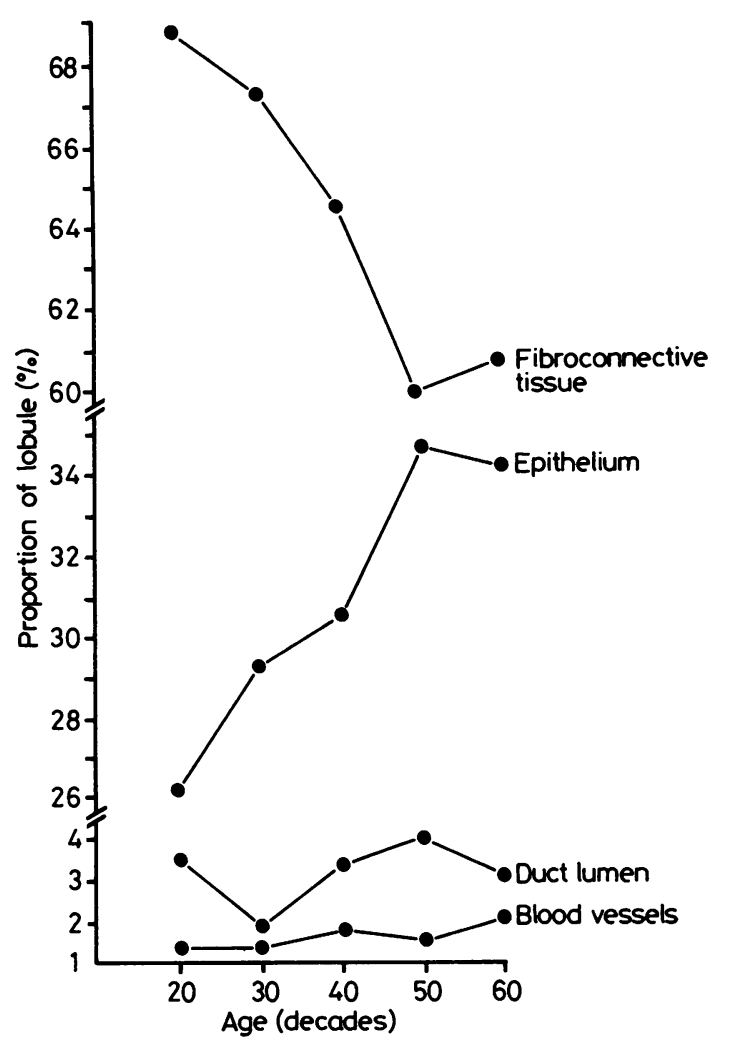

Fig. 6 Composition of average lobule with age.

from nipple to the junction of gland tissue with fat, was recognisable. It was assumed for the purposes of this analysis that there were no interlobe variations in composition within each quadrant. As a result the volume proportions determined for the lobe area were assumed to represent the volume proportions of the average lobe in that quadrant.

The maximum proportion of lobular units was attained in the upper quadrants a decade earlier than the lower quadrants (third versus fourth decade) (Fig. 3). The subsequent decline in volume proportion of lobular units in all quadrants was rapid thereafter except in the upper outer quadrant, which showed a second peak during the fifth decade. Fig. 3 also shows that with the exception of the fourth decade the upper outer quadrant contained the largest proportion of lobular units. During the third to the sixth decades the level of fibrous connective tissue within the average lobe declined (Fig. 4) while the level of fat increased (Fig. 5). During the seventh decade these trends tended to reverse.
COMPOSITION OF AVERAGE LOBULE

Between the second and fifth decades the volume proportion of fibrous connective tissue within lobules decreased while total epithelial cells (acinar and intralobular duct) increased (Fig. 6). Both components showed a slight reversal of this trend during the sixth decade. The volume proportion of lumen (acinar and intralobular duct combined) remained relatively constant except during the third decade, when this value decreased. The volume proportion of blood vessels remained relatively constant.

\section{SIZE AND SHAPE OF AVERAGE LOBULE}

With increasing age the mean diameter and volume of the average breast lobule gradually declined (Figs. 7 and 8). Although the results illustrated assume the lobule to be spherical, a similar trend was apparent when an ellipsoidal shape was assumed. In contrast, the average lobule profile assessed using the form factors $F_{P E}$ and $F_{E L L}$ remained relatively constant $(0.85$ and 0.68 respectively), which suggests that although the size decreased, lobule shape remained unaffected.

\section{EFFECT OF PARITY AND MENOPAUSAL STATE}

The Table shows the parity and menstrual states of cases, where available. No correlation was found between the number of pregnancies and the amount of epithelial tissue present within the breast in any of the different age groups studied (data not shown). The menopause in all patients in this series occurred during the fifth and sixth decades. The proportion of epithelial tissue was greatest during the third decade and declined progressively thereafter before the onset of the menopause (Fig. 2). In postmenopausal subjects the proportion of epithelial tissue remained fairly constant.

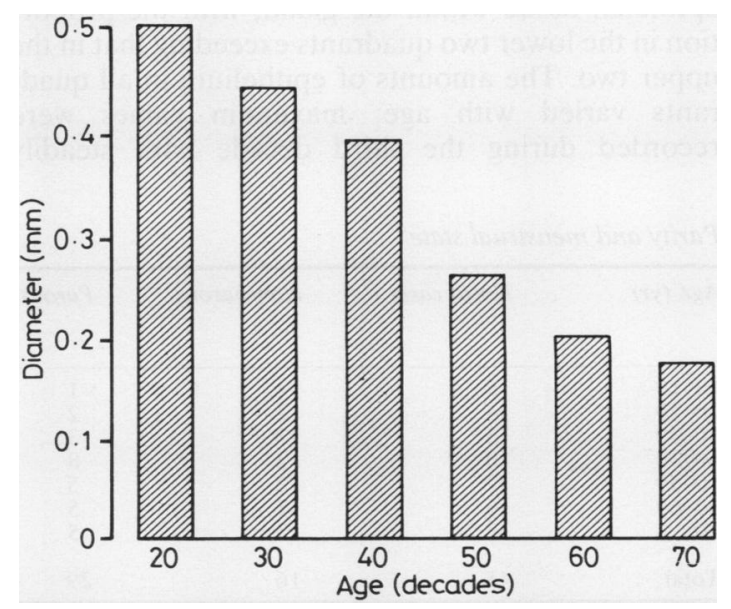

Fig. 7 Changes in mean lobule diameter with age. 


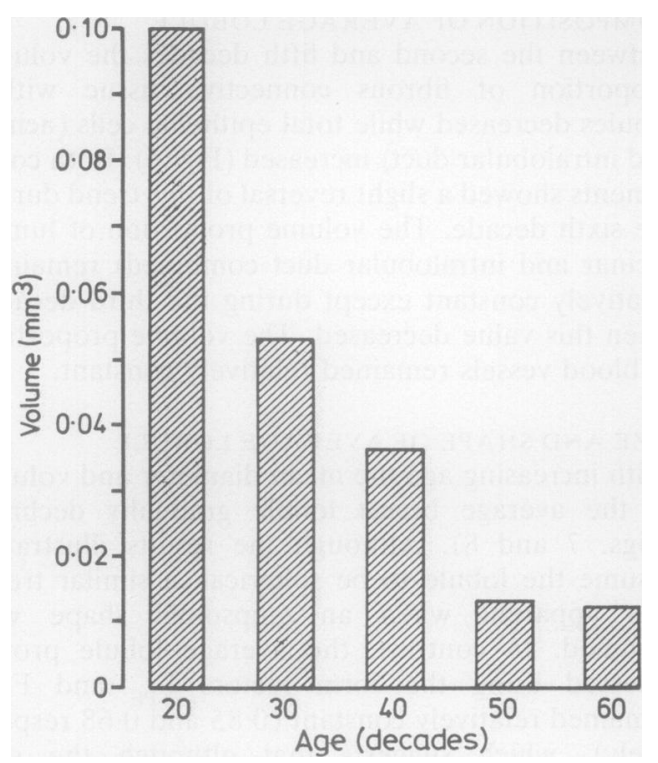

Fig. 8 Changes in mean lobule volume with age.

\section{Discussion}

Although mammary carcinoma develops more frequently in the upper outer quadrant of the mammary gland, ${ }^{9}$ no satisfactory explanation for this polarisation has yet been advanced. Haagensen ${ }^{9}$ suggested that it might be related to increased amounts of epithelial tissue in this quadrant. In an attempt to verify this hypothesis we assessed the volume proportion of epithelial tissue in each quadrant at the subgross level using point counting. The results clearly show an uneven distribution of epithelial tissue within the gland, with the proportion in the lower two quadrants exceeding that in the upper two. The amounts of epithelium in all quadrants varied with age; maximum values were recorded during the third decade with steadily declining values thereafter. These findings do not therefore corroborate Haagensen's hypothesis," although it should be remembered that when assessed at the subgross level no distinction can be made between lobular and extralobular epithelial components. Thus significant variations in the relative proportions of either component might be masked. For this reason a more detailed histological investigation was implemented in an attempt to quantify epithelial and connective tissue elements within the lobe and lobule.

Analysis of the average lobe in each quadrant showed that, with the exception of the fourth decade, the volume proportion of lobular units (epithelial and connective tissue components combined) was greatest in the upper outer quadrant. Since this correlates with the higher incidence of cancer in this quadrant ${ }^{9}$ it seems not unreasonable to infer that the increased proportion of lobular elements somehow predisposes to subsequent neoplastic transformation. As yet, however, there is no explanation as to the pathogenetic mechanisms entailed. Attention was drawn to the importance of the lobule as the site of origin of various breast lesions, including carcinomas, by Wellings et al. ${ }^{10}$ They found that invariably the smallest solitary, independent foci of ductal carcinoma in situ were located in the terminal ductal-lobular units (in the present study referred to as lobules) and not, as previously thought, in the extralobular ducts. To account for the previous observations they suggested that lobular and ductular distension with unfolding and concomitant coalescence produced lobular structures with large ovoid or circular profiles, which in conventional two dimensional histological sections would appear as ducts. Our findings are unable to confirm or refute these suggestions.

Since little or no interquadrant variation was found in either the composition or the size of the breast lobule values for these parameters were pooled and expressed on a whole gland basis. The

Parity and menstrual state*

\begin{tabular}{|c|c|c|c|c|c|c|}
\hline \multirow[t]{2}{*}{ Age (yr) } & \multirow[t]{2}{*}{ No of cases } & \multirow[t]{2}{*}{ Non-parous } & \multirow[t]{2}{*}{ Parous } & \multicolumn{3}{|c|}{ Menopausal state } \\
\hline & & & & Pre- & Peri- & Post- \\
\hline $\begin{array}{l}10-19 \\
20-29 \\
30-39 \\
40-49 \\
50-59 \\
60-69 \\
70\end{array}$ & $\begin{array}{r}5 \\
4 \\
5 \\
10 \\
7 \\
6 \\
8\end{array}$ & $\begin{array}{l}4 \\
2 \\
2 \\
2 \\
2 \\
1 \\
3\end{array}$ & $\begin{array}{l}1 \\
2 \\
3 \\
8 \\
5 \\
5 \\
5\end{array}$ & $\begin{array}{l}5 \\
4 \\
5 \\
6 \\
1 \\
0 \\
0\end{array}$ & $\begin{array}{l}\mathbf{0} \\
\mathbf{0} \\
\mathbf{0} \\
\mathbf{2} \\
\mathbf{0} \\
\mathbf{0} \\
\mathbf{0}\end{array}$ & $\begin{array}{l}0 \\
0 \\
0 \\
2 \\
6 \\
6 \\
8\end{array}$ \\
\hline Total & 45 & 16 & 29 & 21 & 2 & 22 \\
\hline
\end{tabular}

${ }^{*}$ Reliable data available for only 45 of 58 subjects. 
most noticeable change in lobules was the gradual decrease in size with age. In addition, Fig. 6 shows that the proportion of lobular epithelium increases while the fibroconnective tissue decreases. When these figures are expressed in absolute terms, however, we find that the volumes of connective tissue and epithelium reach their maximum during the third decade of life and then decline rapidly. Clearly, this loss of connective tissue and epithelium predates the onset of menopause.

The absolute volumes were calculated by multiplying the lobule volume by the volume proportion of connective tissue and epithelium for each age group. The age at which such changes and the menopause occur indicate that involution of breast tissue begins well before the menopause. No significant morphometric change in lobule shape (as determined by the form factors $F_{P E}$ and $F_{E L L}$ ) occurred with ageing, and our results suggest that the normal lobule profile is slightly elliptical and that the three dimensional lobule shape is more ellipsoidal than spherical.

A notable finding in this study was the relative paucity of changes indicative of microscopic or macroscopic fibrocystic disease or mammary dysplasia in any of the age groups studied. The true.incidence of such changes in the normal breast is difficult to assess accurately particularly since it is impossible to discern the earliest dysplastic changes from normality. In the findings of this study of unselected breasts they appear to be less common than hitherto indicated, ${ }^{1718}$ but in the present study the number of breasts was smaller. The degree of duct ectasia noted was considered to represent a normal feature of the ageing breast. The atypical and cystically dilated lobules of Wellings $e a l^{10}$ and Squartini and Sarnelli" which were found in cancerous and ipsilateral to cancerous breasts would therefore seem to represent distinctive proliferative lesions unrelated to normal ageing changes and indicative of imminent or frank malignant change.

\section{References}

' Geschichter CF. Diseases of the breast. Diagnosis, pathology, treatment. Philadelphia: JB Lippincott Company, 1943.

${ }^{2}$ Bonser GM, Dossett JA, Jull JW. Human and experimental breast cancer. London: Pitman Medical Publishing Co Ltd, 1961.

${ }^{3}$ Ozzello L. Epithelial-stromal junction of normal and dysplastic mammary glands. Cancer 1970;25:586-600.

${ }^{4}$ Ozzello L. Ultrastructure of the human mammary gland. Pathol Ann 1971;6:1-59.

${ }^{5}$ Ozzello L. Electron microscopic study of functional and dysfunctional human mammary glands. J Invest Dermatol 1974;63:19-26.

- Stirling JW, Chandler JA. The fine structure of the normal, resting terminal ductal-lobular unit of the female breast. Virch Arch [A] Pathol Anat Histol 1976;372:205-26.

' Stirling JW, Chandler JA. The fine structure of ducts and subareolar ducts in the resting gland of the female breast. Virch Arch [A] Pathol Anat Histol 1977;373:119-32.

${ }^{8}$ Azzopardi JG. Problems in breast pathology. London: WB Saunders Co Ltd, 1979.

' Haagensen CD. Diseases of the breast. London: WB Saunders Co Ltd, 1971.

${ }^{10}$ Wellings SR, Jensen HM, Marcum RG. An atlas of subgross pathology of the human breast with special reference to possible precancerous lesions. J Natl Cancer Inst 1975;55:231-73.

"Squartini F, Sarnelli R. Structure, functional changes and proliferative pathology of the human mammary lobule in cancerous breasts. J Natl Cancer Inst 1981;67:33-46.

${ }_{12}$ Wellings SR, Jensen HM. On the origin and progression of ductal carcinoma in the human breast. J Natl Cancer Inst 1973; 50: 1111-8.

${ }^{13}$ Delesse MA. Procédé mécanique pour déterminer la composition des roches. CR Acad Sci (Paris) 1847;25:544.

14 Weibel ER. Stereological methods. Vol 1. Practical methods for biological morphometry. London: Academic Press, 1979.

is Aherne WA, Dunnill MS. Morphometry. London: Edward Arnold, 1982.

${ }^{16}$ Fisher RA, Yates F. Statistical tables for biological, agricultural and medical research. 6th ed. Edinburgh: Oliver and Boyd, 1963.

${ }^{17}$ Frantz VK, Pickren JW, Melcher GW, Auchincloss H. Incidence of chronic cystic disease in so-called "normal breasts". Cancer 1951;4:762-83.

${ }^{18}$ Sandison AT. An autopsy study of the adult human breast. National Cancer Institute Monograph no 8. Bethesda, Maryland: US Department of Health, Education and Welfare, 1962.

Requests for reprints to: Dr PN Cowen, Department of Pathology, University of Leeds, Leeds LS2 9JT, England. 